\title{
Prevalence of Hypertensive Disorder of Pregnancy in North India
}

\author{
Seema Kumari \\ Asstt Professor (Physiology) P.M.C.H. Patna
}

\begin{abstract}
Aims and Objective: Hypertensive disorders of pregnancy (HDP) are among the commonest medical disorders during pregnancy constituting one of the greatest causes of maternal and perinatal morbidity and mortality worldwide. This study aimed to analyze the prevalence of hypertensive disorder of pregnancy in North India
\end{abstract}

Material and Method: The study group consisted of a total of 150 pregnant women attending Maharaja Agrasen hospital OPD/IPD during their second trimester (14-20 weeks) of pregnancy from Dec 2016 to Nov 2017.

Results: Out of 146 cases studied , 129 cases remained normotensive and 17 cases developed HDP. Out of 17 cases ,8 cases had mild HDPs and 9 cases had severe HDPs. The prevalence of HDP was 11.7\% in present study.

Conclusion: The study concluded that the incidence of hypertensive disorders in pregnancy was higher than worldwide average and hence early diagnosis and intervention through regular antenatal checkup is pivotal to prevent hypertensive disorders of pregnancy and its complications.

Key words: HDP, Hypertension, Pregnancy, North India

\section{Introduction}

Hypertensive disorders of pregnancy (HDP) are among the commonest medical disorders during pregnancy constituting one of the greatest causes of maternal and perinatal morbidity and mortality worldwide. ${ }^{1-4}$ HDP complicate up to $5-10 \%$ of all pregnancies. ${ }^{5}$ It accounts for $10-15 \%$ of maternal deaths specially in the developing world. ${ }^{6}$ The spectrum of disease ranges from mildly elevated blood pressures with minimal clinical significance to severe hypertension and multi-organ dysfunction .Besides perinatal death ,HDP lead to preterm delivery, fetal intrauterine growth restriction, low birth weight. HDP can also trigger severe

\footnotetext{
Corresponding Author:

Dr Seema Kumari

Dr Seema Kumari, Asstt Professor (Physiology), P.M.C.H. , Patna, North Patel Nagar, Patna 800024

E-mail: drseemakumari2@gmail.com
}

forms of maternal complications, such as cardiovascular and cerebrovascular diseases, liver and kidney failure, placental abruption, disseminated intravascular coagulation (DIC) and HELLP syndrome.

Hypertension in pregnancy is defined as a systolic $\mathrm{BP}$ of $140 \mathrm{mmHg}$ and higher, and a diastolic BP of 90 $\mathrm{mmHg}$ and higher. Severe pregnancy hypertension is defined as SBP $\geq 160 \mathrm{mmHg}$ or a $\mathrm{DBP} \geq 110 \mathrm{mmHg}$. The systolic value was reduced from $170 \mathrm{mmHg}$ by most international societies after recognition that a $\mathrm{SBP} \geq 160$ $\mathrm{mmHg}$ is associated with an increased risk of stroke in pregnancy.

Hypertensive disorders during pregnancy are classified into four categories, as recommended by the National High Blood Pressure Education Program (2000) Working Group on High Blood Pressure in Pregnancy ${ }^{7}$ :

- Gestationalhypertension(transienthypertension of pregnancy)

- $\quad$ Preeclampsia-eclampsia 
- Preeclampsia superimposed on chronic hypertension

- Chronic hypertension

Aims \& Objective: To find the prevalence of Hypertensive disorder of pregnancy in North India.

\section{Material and Method}

Study site: Maharaja Agrasen Hospital, Punjabi Bagh, New Delhi.

Study population: Woman with singleton pregnancy visiting Obs \& Gynae OPD/IPD of Maharaja Agrasen hospital, Punjabi Bagh during their second trimester (1420 weeks) of pregnancy

Study Design : Prospective observational study

Sample size: 150

\section{Inclusion Criteria}

- 1.All pregnant women in their second trimester (14 - 20 weeks) above 18 years and below 40 years of age with informed consent.

- 2. Pregnancy confirmed by ultrasonography

- 3. Singleton pregnancy

- 4. Previously normo tensive and non proteinuric

\section{Exclusion Criteria}

The established exclusion criteria are as follows:

- Age less than 18 and more than 40 years

- Multiple pregnancy

- Chronic Hypertension

- Gestational trophoblastic diseases in present or previous pregnancy
- Down syndrome/any congenital anomalies

- Pregnancy associated with Germ cell tumors

- Diabetes mellitus

\section{Methodology}

- A total of 150 pregnant women attending Maharaja Agrasen hospital OPD/IPD during their second trimester (14-20 weeks) of pregnancy were enrolled in the study.

Diagnostic criteria for Gestational Hypertension was new onset of hypertension $(\geq 140 \mathrm{mmHg}$ systolic and/or $\geq 90 \mathrm{mmHg}$ diastolic) after 20 weeks gestation without Proteinuria and signs of end organ dysfunction.

Diagnostic criteria for Pre-eclampsia (revised ISSHP, 2014) was Hypertension after 20 weeks gestation and the coexistence of one or more of the following new-onset conditions:

1. Proteinuria (spot urine protein/creatinine $>30 \mathrm{mg} /$ $\mathrm{mmol}[0.3 \mathrm{~g} / \mathrm{mg}]$ or $>300 \mathrm{mg} /$ day or at least $1 \mathrm{~g} / \mathrm{L}\left[{ }^{6} 2+\right.$ '] on dipstick testing).

2. Other maternal organ dysfunction: renal insufficiency (creatinine $>90 \mathrm{umol} / \mathrm{L} ; 1.02 \mathrm{mg} / \mathrm{dL}$ ), liver involvement (elevated transaminases - at least twice upper limit of normal \pm right upper quadrant or epigastric abdominal pain), neurological complications ( eclampsia, altered mental status, blindness, stroke,or more commonly hyperreflexia when accompanied by clonus, severe headaches when accompanied by hyperreflexia, persistent visual scotomata), haematological complications (thrombocytopenia, , DIC, haemolysis).

3. Uteroplacental dysfunction (foetal growth restriction)

Diagnostic criteria for Eclampsia was Preeclampsia associated with convulsion.

\section{Observation \& Results}

Table 1: Mean Age of the three groups (Normotensive, Mild HDP and Severe HDP)

\begin{tabular}{|l|l|l|l|l|}
\hline GROUPS & MEAN +/_SD & RANGE & MEDIAN & \multirow{2}{*}{ P VALUE } \\
\cline { 1 - 3 } NORMOTENSIVE (B) & $27.86+/-3.71$ & $19-37$ & 28 & \multirow{2}{*}{0.10} \\
\cline { 1 - 3 } MILD HDP( A1) & $29.87+/-4.63$ & $21-38$ & 30 & \\
\hline SEVERE HDP (A2) & $29.11+/-4.40$ & $24-39$ & 29 & \\
\hline
\end{tabular}




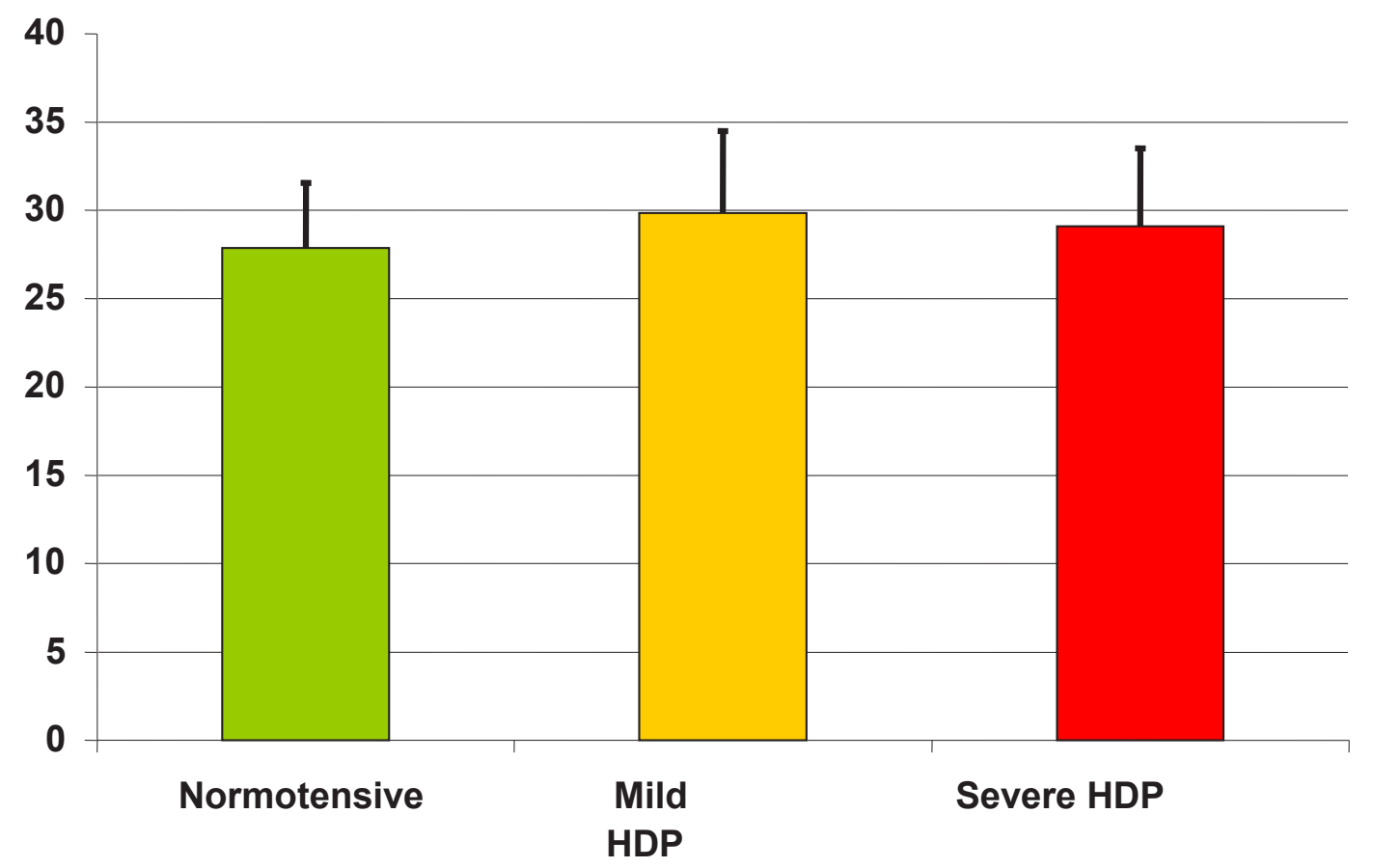

$\square$ Normotensive $\square$ Mild HDP $\quad \square$ Severe HDP

The mean age of normotensive group was 27.86+/-3.71, whereas in Mild HDP and Severe HDP group was $29.87+/-4.63$ and $29.11+/-4.40$. There was statistically no significant difference between age of three groups.

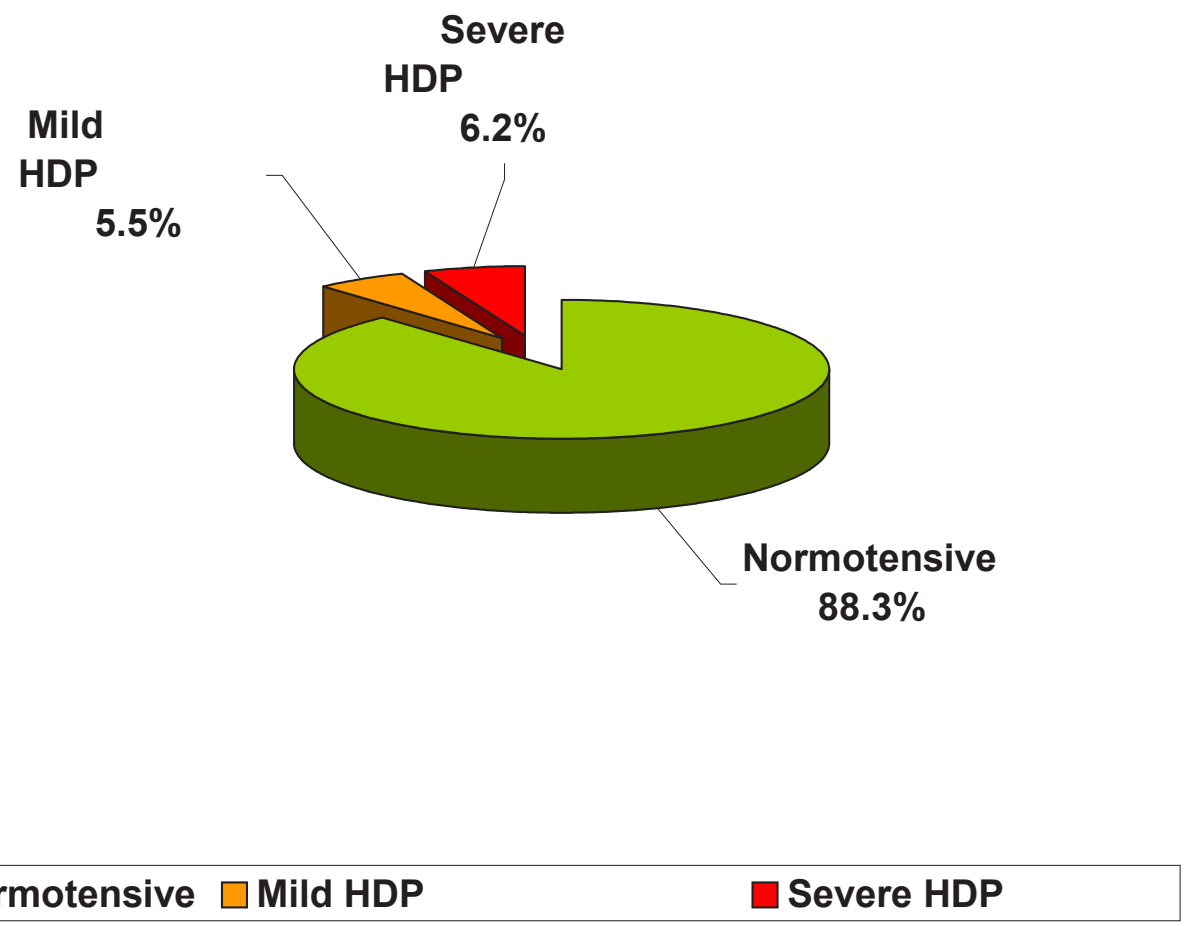

Corrected Chi-square () test ( $=13.28 ; \mathrm{p}=0.10$ NS-Not Significant) showed that there was no significant association between age and cases of the three groups $(\mathrm{p}=0.10)$. Thus the cases of the three groups were more or less equally distributed over age. 
One ANOVA showed that there was no significant difference between the mean age of the cases of the three groups $\left(\mathrm{F}_{2,143}=2.11 ; \mathrm{p}=0.12\right)$. Thus the cases of the two groups were age matched.

Table-2: Prevalence of HDP

\begin{tabular}{|l|l|l|}
\hline Group & Number & $\mathbf{\%}$ \\
\hline Normotensive & 129 & $88.4 \%$ \\
\hline Mild HDP & 8 & $5.5 \%$ \\
\hline Severe HDP & 9 & $6.2 \%$ \\
\hline Total & 146 & $100.0 \%$ \\
\hline
\end{tabular}

Out of the 146 cases under study 129(88.4\%) cases were normotensive, $8(5.5 \%)$ were Mild HDP and rest $9(6.2 \%)$ were severe HDP. In overall $17(11.7 \%)$ were HDP. Thus the prevalence of HDP was $11.7 \%$.

\section{Discussion}

Hypertensive disorders of pregnancy (HDP) is a major challenge in overcoming pregnancy complications that are responsible for poor maternal and prenatal outcome in developed as well as underdeveloped countries of the world. These disorders comprise of chronic hypertension, gestational hypertension, preeclampsia and eclampsia.

The spectrum of HDP ranges from mildly elevated blood pressures with minimal clinical significance to severe hypertension and multi-organ dysfunction.

In my study, 150 cases were initially enrolled. However, only 146 cases (97.3\%) could be evaluated for the final results. The 4 cases were lost to follow up.

These cases were divided into two groups:

- Group A - who developed HDP.

- Group B - who remained normotensive

Group A was divided further into two group i.e. Group A1 and Group A2 based on features of severity. Cases in Group A1 were mild hypertensive and A2 were severe hypertensive.

The mean age of the cases for my study was 27.86 (Group B normotensive) 29.87 (Group A1, mild hypertensive) and 29.11 (Group A2, severe hypertensive).
There was no statistically significant correlation found between the age and the occurrence of HDP which was in concordant with the results of study conducted by Vishal Sharma et al (2016) ${ }^{7}$, who observed that there was no statistically significant difference between age of subjects and HDP. $76.34 \%$ of the patients in my study were between 21 and 30 years of age thus rendering a very young population morbid and at risk of mortality.

The prevalence of HDPs observed in my study is 11.7 $\%$. Incidence of hypertensive disorders in India is found to be $10.08 \%$ as observed through the data collected by the National Eclampsia Registry (NER) (11,266 out of $1,11,725$ deliveries) over the 3 consecutive years ${ }^{8,9}$. The prevalence matches out with NER data considering the fact that my study place is tertiary care centre where high risk patients having more visits.

In a study conducted by Vidyabati R K et al $(2010)^{10}$ prevalence rate of HDPs was $17.68 \%$. In the study by Charu sharma et al (2017) ${ }^{11}$, the incidence of HDP came out to be $6.92 \%$. The prevalence of hypertension during pregnancy was found to be $6.9 \%$ in the study conducted by Bharti Mehta et al $(2015)^{12}$. Hypertensive disorders of pregnancy were reported to be $15.5,5.38$, and $8.96 \%$, respectively, in other various hospital-based studies in India. ${ }^{13,14,15}$

\section{Summary \& Concusion}

In my study, 150 cases were initially enrolled. However, only 146 cases $(97.3 \%$ ) could be evaluated for the final results. The 4 cases were lost to follow up . Out of 146 cases studied , 129 cases remained normotensive and 17 cases developed HDP. Out of 17 cases , 8 cases had mild HDPs and 9 cases had severe HDPs. The prevalence of HDP was $11.7 \%$ in my study.

The mean age of the cases for my study was 27.86(Group B normotensive ) 29.87 (Group A1, mild hypertensive ) and 29.11 (Group A2, severe hypertensive ). There was no statistically significant correlation found between the age and the occurrence of HDP.

Acknowledgement: Author would like to thank Dr Angela Sehra, Senior consultant \& Dr Seema Bhardwaj, Consultant Maharaja Agrasen Hospital, Punjabi Bagh, New Delhi

Ethical Clearance- Taken

Source of Funding- Self 


\section{Conflict of Interest - Nil}

\section{References}

1. Alessia Mammaro, Sabina Carrara. Hypertensive Disorders in Pregnancy Journal of Prenatal Medicine 2009; 3 (1): 1-5

2. Bombrys AE, Barton JR, Habli M et al. Expectant management of severe preeclampsia at $27(0 / 7)$ to 33(6/7) weeks' gestation: maternal and perinatal outcomes according to gestational age by weeks at onset of expectant management. Am J Perinatol 2009;26:441-46.

3. Berg CJ, Chang J, Callaghan WM. Pregnancyrelated mortality in the United States 1991-1997. Obstet Gynaecol. 2003;101(2):289-96.

4. Berg CJ, Harper MA, Arkinson SM. Preventability of pregnancy related deaths. Obstet Gynaecol. 2005;106(6):1228-34.

5. Hypertensive disorders, Williams obstetrics, 24 edition, page 728-779

6. Vigil-De Gracia P, Montufar-Rueda C, Ruiz J.Expectatnt management of severe preeclampsia and preeclampsia superimposed on chronichypertension between 24 and 34 weeks gestation. Eur J Obstet Gynecol Reprod Biol 2003;107(1): 24-7.

7. Vishal Sharma, Preeti Sharma,Nighat Firdous BETA HCG in Mid Trimester as a Predictor of Pregnancy Induced Hypertension International Journal of Science and Research (IJSR) Volume 5 Issue 9, September 2016.
8. http://www.ner-fogsi.in/

9. Gupte Sanjay, Wagh Girija . PreeclampsiaEclampsia ; The Journal of Obstetrics and Gynecology of India (January-February 2014) 64(1):4-13

10. Vidyabati RK, Davina H, Singh NK, et al. Serum $\beta$ hCGlevels and lipid profile in early second trimester as predictors of pregnancy induced hypertension. $\mathrm{J}$ Obstet Gynecol India. 2010;60(1):44-50. doi: 10.1007/s13224-010-0008-1.

11. Charu Sharma, Smriti Gupta, Mamta Tyagi ; Maternal \& Perinatal outcome in Hypertensive Disorders of Pregnancy in a Tertiary Care Hospital in Northern India; OGIJ 0600229 Volume 6 issue 62017

12. Mehta B, Kumar V, Chawla S, Sachdeva S, Mahopatra D. Hypertension in pregnancy: A community-based study. Indian J Community Med [serial online] 2015

13. Mohan BS. Pregnancy induced hypertension and prior trophoblastic exposure. J Obstet Gynecol Ind 2004;54:568-70.

14. Prakash J, Pandey LK, Singh AK, Kar B. Hypertension in pregnancy: Hospital based study. J Assoc Physicians India 2006;54:273-8.

15. Bangal VB, Giri PA, Mahajan AS. Maternal and foetal outcome in pregnancy induced hypertension: A study from rural tertiary care teaching hospital in India. Int $\mathrm{J}$ Biomed Res 2011;2:595-9. 